\title{
Factors Associated with Diabetes- Related Distress in Patients with Type 2 Diabetes Mellitus
}

\author{
Karla Duka1, Corina Roxana Onea', Simona Cernea 2,3 \\ 1 Emergency County Clinical Hospital, Târgu Mureş, Romania \\ 2 Department M3, Internal Medicine I, "George Emil Palade” University of Medicine, Pharmacy, Science and Technology, Târgu Mureş, \\ Romania \\ 3 Diabetes, Nutrition and Metabolic Diseases Outpatient Unit, Emergency County Clinical Hospital, Târgu Mureş, Romania
}

\section{CORRESPONDENCE}

\section{Simona Cernea}

Str. Gheorghe Marinescu nr 38

540139 Târgu Mureş, Romania

Tel: +40265215551

E-mail: simona.cernea@umfst.ro;

simonacernea@yahoo.com

\section{ARTICLE HISTORY}

Received: June 19, 2021

Accepted: June 30, 2021
Karla Duka • Str. Gheorghe Marinescu nr. 50, 540138 Târgu Mureș, Romania. Tel: +40 265212 111, E-mail:

Corina Roxana Onea $\cdot$ Str. Gheorghe Marinescu nr. 50, 540138 Târgu Mureş, Romania. Tel: +40 265212 111, E-mail:

\begin{abstract}
Introduction: The aim of this study was to identify factors associated with diabetes-related distress (DRD) in adult patients with type 2 diabetes mellitus (T2DM). Material and Methods: This was an analysis of data previously obtained from two cross-sectional studies, in which medical charts review and direct interviews were employed to obtain medical and demographic data. Vital status assessment and anthropometric measurements were performed. The patients filled out specific questionnaires for DRD (DDS-17), symptoms of depression (PHQ-9), and of anxiety (GAD-7). A clinical meaningful threshold for DRD was set at $\geq 2.0$ points. Symptoms of depression and anxiety, number of chronic complications, therapy for T2DM, anthropometric and cardiometabolic parameters, as well as demographic, socio-economic data, and lifestyle habits were evaluated as factors possibly associated with DRD by univariate and multiple regression analyses. Results: A total of 271 patients with T2DM were included in this analysis, of whom $25.1 \%$ presented a DDS-17 score $\geq 2$ points (and 9.96\% a DDS-17 score $\geq 3$ ). Subjects with a DDS-17 score $\geq 2$ had higher HbA1c levels ( $p=0.018)$, PHQ-9 and GAD-7 scores ( $<0.0001$ for both). The multiple regression model indicated that anxiety $(p=0.026)$, depression $(p=0.001)$, and ethnicity $(p=$ 0.002 ) were significantly correlated with DRD ( $p<0.0001)$. With regards to subscales, the HbA1c $(p=0.005)$ and PHQ-9 score $(p<0.0001)$ were significantly associated with emotional burden, ethnicity $(p=0.001)$ and depression $(p=0.004)$ with regimen-related distress, whereas ethnicity $(p=0.010)$ and GAD-7 score $(p=0.012)$ with interpersonal distress. Conclusions: Psychosocial factors like depression, anxiety, or ethnicity significantly contribute to DRD in patients with T2DM, and worse glycemic control is associated with emotional burden.
\end{abstract}

Keywords: type 2 diabetes mellitus, diabetes-related distress, depression, anxiety

\section{INTRODUCTION}

Type 2 diabetes mellitus (T2DM) is a chronic metabolic disease that requires lifelong, comprehensive care, and is frequently associated with neuropsychological comorbidities, such as depression, anxiety, or cognitive impairment, 
conditions that may affect the metabolic control, diabetes self-care behaviors including medication adherence, and quality of life. ${ }^{1-5}$

In addition, it is apparent that a high proportion of T2DM patients also present diabetes-related distress (DRD), defined as psychological reactions or emotions that they might experience. These psychological reactions are basically concerns related to disease management, perception of support, access to quality healthcare, or emotional burden. ${ }^{6}$ A systematic review and meta-analysis of 55 studies ( $\mathrm{n}=36,998$ subjects) indicated that $36 \%$ of individuals with T2DM suffer from DRD (but with very large variations, ranging from to about $10 \%$ to over $60 \%) .^{7-9}$ The difference may be due to patient selection, geographical area/ ethnicity, method of assessment and interpretation, or the presence of comorbid conditions, such as coexistence of depression, which increases the prevalence of DRD. ${ }^{7}$

Female gender and depression were the only factors identified in the meta-analysis of Perrin $\mathrm{et} \mathrm{al}$. as being associated with DRD, but a plethora of other factors emerged from the literature such as age, body mass index (BMI), glycemic control, duration of diabetes, lifestyle habits, treatment regimens, level of education etc.,10-16

DRD has important health-related consequences such as worse glycemic outcomes, poorer medication adherence and diabetes self-care, and poorer quality of life..$^{5,17} \mathrm{~A}$ recent study reported that in patients with T2DM, depressive symptoms were associated with lower self-management behavior and higher glycated hemoglobin (HbAlc), while DRD was associated with higher HbAlc, suggesting that patients with both depression and DRD might have worse outcomes. ${ }^{18}$

During recent years, there has been an increasing interest regarding the association between T2DM and DRD, yet currently there are scarce data regarding DRD in patients with T2DM from Romania.

The aim of this study was to identify factors that have a significant impact on DRD in adult patients with T2DM, by exploring a number of demographic, socio-economic, and lifestyle factors, cardio-metabolic parameters, type of treatment, and chronic complications.

\section{MATERIAL AND METHODS}

This was a post-hoc analysis of data obtained in two crosssectional studies that screened adult patients with T2DM for symptoms of depression and anxiety, and for DRD using specific questionnaires, in 2015, and between 2018 and 2019, respectively. Each of the studies were approved by the ethics committees of the Emergency County Clinical
Hospital of Târgu Mureş and of the "George Emil Palade" University of Medicine, Pharmacy, Science and Technology of Târgu Mureş, and patients signed an informed consent before participating. Patients were recruited from two specialty-care settings, the Diabetes, Nutrition and Metabolic Diseases Outpatient Unit of the Emergency County Clinical Hospital in Târgu Mureş (one specialist physician) and from the Puls Medical Center in Târgu Mureș (two specialist physicians).

The two studies had similar inclusion and exclusion criteria and design. ${ }^{1}$ The included patients were aged $>18$ years, diagnosed with T2DM according to the American Diabetes Association criteria. ${ }^{19}$ Main exclusion criteria were patients with type 1 diabetes mellitus, secondary diabetes mellitus, and gestational diabetes mellitus, severe psychiatric disorders, or unable to read Romanian language.

Both studies collected demographic and socio-economic data obtained from the medical charts and from a direct interview (age, gender, residency, education level, economic level, ethnicity, occupation, marital status), medical history (diabetes duration, micro- and macrovascular complications of diabetes, medication for T2DM and other comorbidities), information regarding lifestyle (level of physical exercise, smoking, alcohol, and coffee intake). Additionally, basic anthropometric parameters (weight, height, abdominal circumference), as well as heart rate and blood pressure measurements were performed, all by standard procedures. The BMI was calculated as weight $(\mathrm{kg}) /$ height $^{2}\left(\mathrm{~m}^{2}\right)$.

In the first study, 145 of the 216 included T2DM patients had laboratory testing performed by drawing blood during the study, while for the remaining, the laboratory information was collected from their medical charts (and included in this analysis only if they were available in the last three months). ${ }^{1}$ For patients enrolled in the second study (20182019), laboratory tests (HbA1c, fasting blood glucose, and basic biochemistry) were collected from medical charts (if results from the last three months were available).

The subjects completed three questionnaires translated to Romanian. DRD was evaluated using the 17-item Diabetes Distress Screening Scale (DDS-17) questionnaire. DDS-17 is one of the two well-accepted tools for evaluation of DRD. It consists of 17 questions, each having assigned between 1 to 6 points. A score $\geq 2$ points for DRD was considered positive (and interpreted as mild DRD), while a score $\geq 3$ points was considered as significant DRD. ${ }^{13,20,21}$ DDS- 17 consists of 4 subscales, each evaluating a different aspect: emotional burden (EB), physicianrelated distress $(\mathrm{PD})$, regimen-related distress $(\mathrm{RD})$, and interpersonal distress (ID). The interpretation of each subscale was performed in a similar manner as for general 
score. The symptoms of depression and anxiety were assessed by the Patient Health Questionnaires-9 (PHQ-9) and Generalized Anxiety Disorder-7 (GAD-7) questionnaires, respectively. For the PHQ-9 questionnaire, a score of $\geq 5$ points indicated mild depression, $\geq 10$ points moderate depression, and $\geq 15$ points severe depression.1,22 For the GAD-7 questionnaire, a score of $\geq 5$ points indicated mild anxiety, $\geq 10$ points moderate anxiety, and $\geq 15$ points severe anxiety. ${ }^{1,23}$

\section{Statistical analysis}

Continuous variables with a normal distribution were presented as mean (standard deviation [SD]), while those non-normally distributed as median (min-max), and categorical variables as frequency (\%). The KolmogorovSmirnov test was used to determine whether the data had a normal distribution. For categorical variables, Fisher's exact test was used, and the odds ratios (OR) were calculated. Student's t test or the Mann-Whitney test was used to compare the differences between groups, and the bivariate relationships between variables were evaluated by Spearman's test (correlation coefficients presented as $\mathrm{r}(95 \% \mathrm{CI}$ [confidence interval]). In order to identify independent associations between DRD and each domain with relevant parameters, we have performed a hierarchical multiple regression model, separately for the total DRD score and for each subscale. Model 1 included demographic, socioeconomic, and medical variables that were identified in the bivariate analysis as being significantly associated with DRD. Model 2 additionally included PHQ-9 and GAD-7 scores, to fully adjust for depression and anxiety. We have chosen these two conditions, as data in the literature and our previous partial data indicated them as being significant for DRD.

All tests were two-tailed, and the statistical significance was set at $\mathrm{p}<0.05$. Statistical analysis was performed using GraphPad InStat3.

\section{RESULTS}

From the two studies, a total of 316 patients with T2DM were included in this analysis. Duplicate examinations and patients lacking laboratory data in the last three months were excluded. In the end, data from 271 patients were analyzed. Table 1 presents the demographic, clinical, and metabolic characteristics of patients with T2DM with and without DRD (a DDS-17 score of $\geq 2$ points).

T2DM patients with clinically meaningful DRD had a better economic level, were rather of Romanian ethnicity, and had higher GAD-7 and PHQ-9 scores, as well as higher HbA1c values (Table 1). No other statistically significant differences were noted between the two groups.

In this pooled population, $25.1 \%$ of $\mathrm{T} 2 \mathrm{DM}$ patients presented a DDS-17 score $\geq 2$ (and $9.96 \%$ had a score $\geq 3$ ). Not only the PHQ-9 and the GAD-7 scores were higher in the DRD group (Table 1), but more T2DM patients with DRD presented more severe degrees of both anxiety and depression (Figure 1). Also, significantly more patients with both PHQ-9 and GAD-7 scores $\geq 10$ points had a DDS-17 score $\geq 2$ points compared with those with negative scores $(<5$ points) (OR 8.91, 95\% CI 3.77 to $21.08, \mathrm{p}<0.0001$ ). In addition, T2DM patients that presented both a PHQ-9 and a GAD-7 score $\geq 10$ points (moderate/severe depression and anxiety, respectively) had a significantly higher DDS17 score than those with both scores $<5$ points (negative for depression and anxiety) (2.11 [min: 1.06; max: 4.53] vs. 1.24 [min: 1.0; max: 3.76], $\mathrm{p}<0.0001)$.

With regards to the DRD domains, in this pooled population a higher prevalence was observed for EB (38.74\%) and RD (40.59\%), while PD was noted in $8.48 \%$, and ID in $19.18 \%$ of the T2DM patients. Moreover, T2DM patients with both moderate/severe depression and anxiety had a significantly higher score for EB (2.6 [min: 1.0; max: 6.0] vs. 1.2 [min: 1.0; $\max : 4.6$ ], p <0.0001), RD (2.3 [min: 1.0; $\max : 5.2]$ vs. 1.4 [min: 1.0; $\max : 4.0], \mathrm{p}<0.0001)$ and ID (1.33 [min: 1.0; max: 6.0] vs. 1.0 [min: 1.0 ; $\max : 5.67$ ], $\mathrm{p}=$ 0.002 ) compared with patients with both negative scores.

The bivariate analysis indicated significant positive correlations between the DDS-17 score and BMI, heart rate, HbAlc, and fasting blood glucose, and negative correlations with age, ethnicity, and occupation (Table 2). The EB scores were negatively correlated with age and occupation, and positively with $\mathrm{HbAlc}$, the PD scores were inversely correlated with ethnicity and positively correlated with heart rate, while the RD scores were positively correlated with HbAlc levels and fasting blood glucose, and negatively with age and occupation. In addition, anxiety and depression scores were positively associated with the total DDS-17 score and with all four subscales. For the rest of the parameters (presented in Table 1), there were no statistically significant correlations with none of the scores (data not shown). The strongest correlations of GAD-7 and PHQ-9 scores were observed with EB and with overall DRD, while all other correlations were weaker (Table 2).

In order to evaluate which of the variables have an independent, significant impact on DRD, we have further performed a stepwise multivariate regression analysis for the total DDS-17 score and each domain separately, by using variables that showed a significant correlation in 
TABLE 1. Demographic, clinical and metabolic characteristics of patients with T2DM without and with DRD

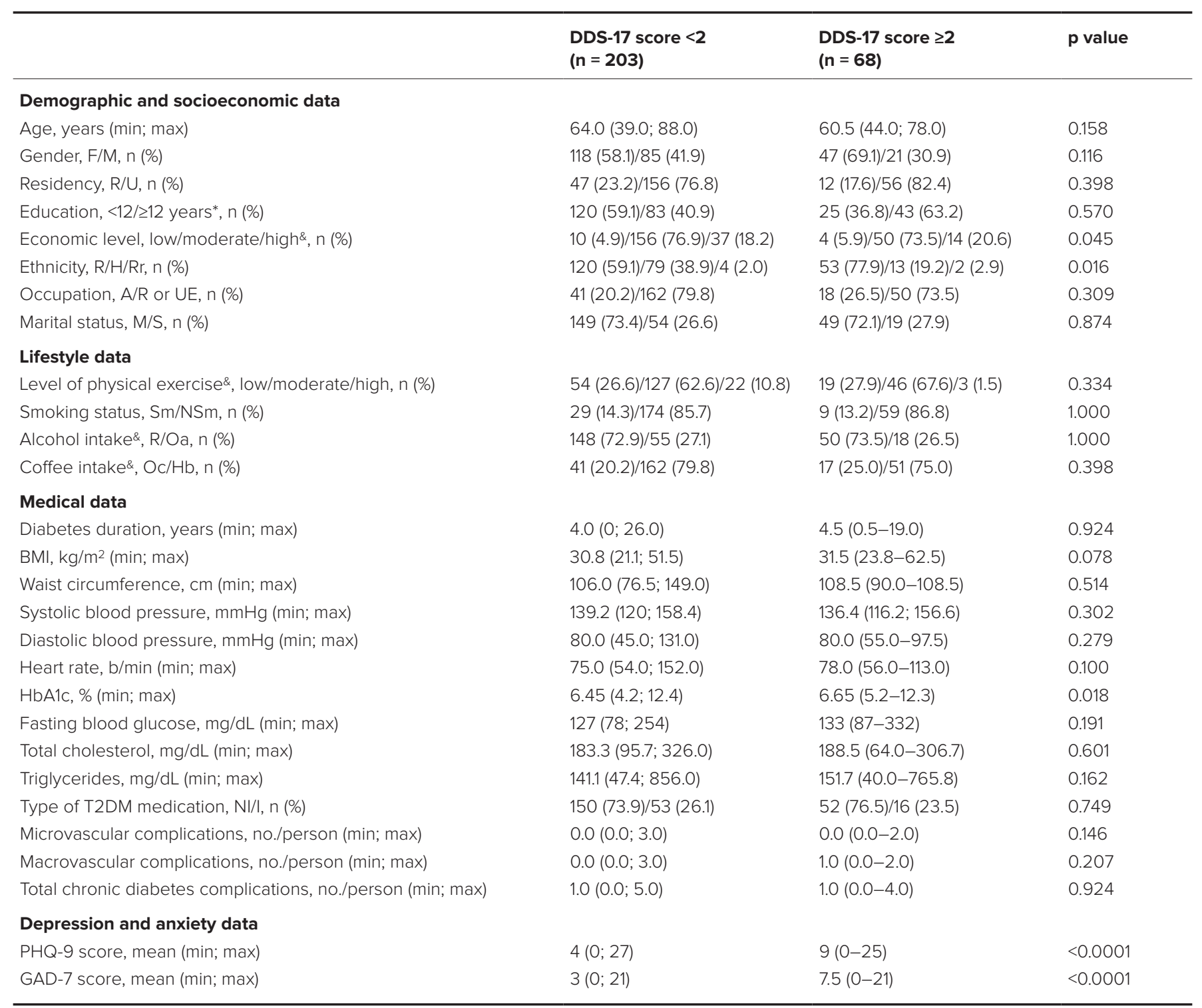

F - female; M - male; $\mathrm{nr}$ - number; R - rural; U - urban; "years of formal education; \&self-declared; R - Romanian; H - Hungarian; Rr - Rroma; A - active; R - retired; UE - unemployed; M married (or living with a partner); S - single (not married/widowed/divorced); Sm - smoker; NSm - non-smoker/ex-smoker; R - regular (daily/weekly); Oa - occasionally (< one time/week); Oc occasionally ( weekly or more rare); $\mathrm{Hb}$ - habitual (at least one daily); BMI - body mass index; b/min - beats/minute; HbA1c - glycated hemoglobin; NI - non-injectable; I injectable

the bivariate analysis (Table 3). The first model included demographic, socio-economic, and medical parameters (age, fasting blood glucose, HbA1c, heart rate, BMI, ethnicity, and occupation), and in the second model GAD-7 and PHQ-9 scores were added.

In model 1 , the BMI was positively (but weakly) correlated with the total DDS-17 score $(\mathrm{p}=0.0387), \mathrm{EB}(\mathrm{p}=$ $0.028)$, and $\mathrm{RD}(\mathrm{p}=0.003)$, while HbA1c was correlated with EB ( $p=0.001)$ (Table 3$)$. Ethnicity significantly associated with the overall DRD ( $p=0.0125), R D(p=0.005)$, and ID $(p=0.026)$. The Hungarian group had a lower overall DRD score (median: 1.29 [1.00; 4.53]) compared to the Romanian group (median: 1.53 [1.00; 5.18]; $\mathrm{p}<0.05$ ), sim- ilarly to the Rroma population (median: 1.44 [1.00; 3.59]); $\mathrm{p}=0.018$ ). Similar results were observed for RD across the three ethnicity groups (median: $1.40[1.00 ; 5.20]$ vs 1.80 $[1.00 ; 5.80], \mathrm{p}<0.05$, and 1.70 [1.00; 4.80]; $\mathrm{p}=0.034$ ).

After full adjustment, the GAD-7 score was significantly correlated with the overall DRD $(\mathrm{p}=0.0267)$ and ID $(\mathrm{p}=$ $0.0123)$, while the PHQ-9 score with the overall DRD ( $\mathrm{p}=$ $0.0013), \mathrm{EB}(\mathrm{p}<0.0001)$, and RD $(\mathrm{p}=0.0043)$ (Table 3$)$.

\section{DISCUSSIONS}

Being diagnosed with diabetes can be challenging, in part because of the long-term healthcare demands, and because 

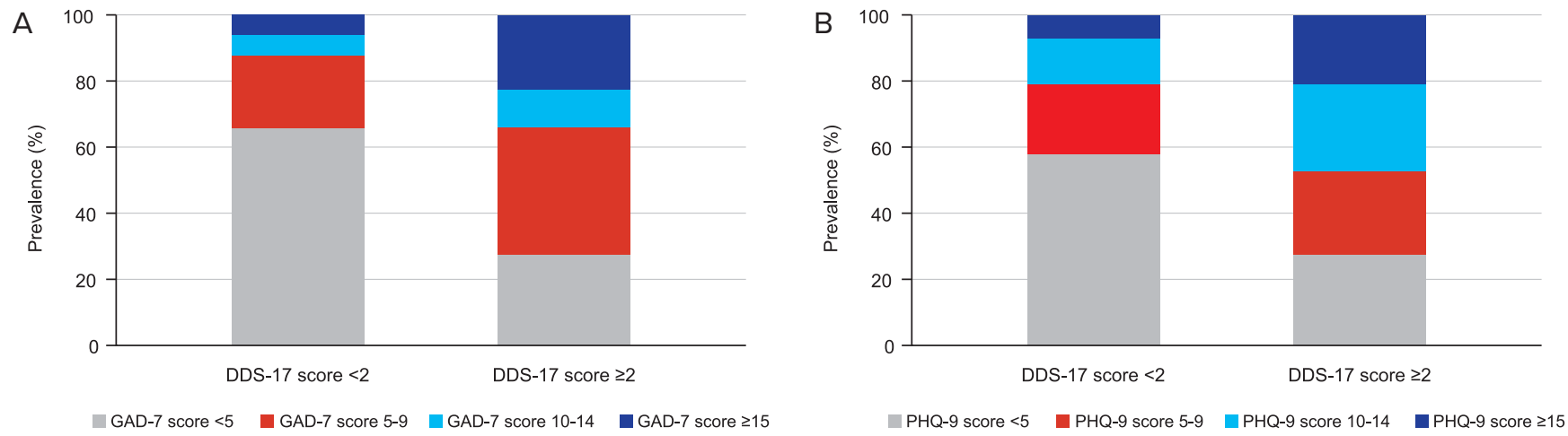

FIGURE 1. Prevalence of generalized anxiety (A) and depression (B) symptoms (stratified by the degree of severity) in patients with and without DRD

of the progressive nature of the disease, which requires permanent check-ups, adjustments of lifestyle habits, pluri-medication etc. Therefore, it is plausible for someone to develop emotional distress related to the illness, followed by lack of motivation and failure. This study is drawing attention upon the need for psychological evaluation and support for persons with T2DM.

It appears that individuals that present DRD are prone to inadequate glycemic control, and this might be related to reduced interest in a healthy lifestyle, but also poor treatment adherence. $11,24,25$ This study results also indicated that patients with DRD had higher HbAlc values. The bivariate analysis showed that both the emotional burden and regimen-related distress positively associated with HbAlc. Additionally, regimen-related distress was positively correlated with fasting blood glucose, although after full adjustment in the multivariate analysis the correlation remained significant for emotional burden. Thus, our study suggests that worse glycemic control is associated rather with emotional distress than poor interpersonal relationships, including with the physician, lack of access to healthcare support, or diabetes regimen. In other words, psychological well-being is an important condition for glycemic control. The question that arises regarding the nature of this interdependence, whether having DRD influences the glycemic outcomes or, on the contrary, higher glycemic values determine certain degrees of emotional distress. The situation is still debatable, and it would need longitudinal evaluation to be clarified. An intervention study in a tertiary-care setting showed a reduction in DRD after a problem-oriented intervention in patients with T2DM, which was also associated with an improvement in metabolic outcomes (HbAlc, blood glucose, insulin units per day, BMI). ${ }^{26}$ However, a Cochrane systematic review that included 30 randomized controlled trials ( $n=9,177$ participants) concluded that psychological intervention did not significantly influence DRD more than usual care. However, the authors suggested a small beneficial effect on self-efficacy and HbAlc (although the quality of evidence was low). ${ }^{27}$ A more recent meta-analysis (8 studies, 841 subjects) on the other hand, reported the beneficial effects of mindfulness-based intervention

TABLE 2. The bivariate correlations of the total DDS-17 score and subscales with variables of interest

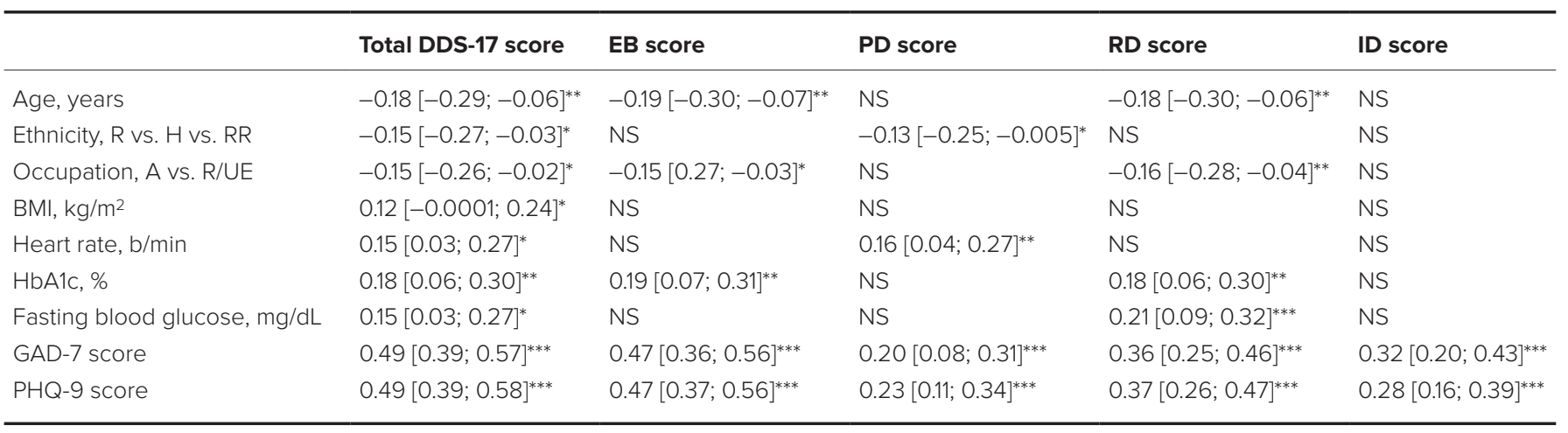

Data are presented as r [95\% Cl]. DDS-17 - Diabetes Distress Scale 17; EB - emotional burden; PD - physician-related distress; RD - regimen-related distress; ID - interpersonal distress; R Romanian; H - Hungarian; RR - Rroma; A - active; R - retired; UE - unemployed; BMI - body mass index; HbA1c - glycated hemoglobin; GAD-7 - General Anxiety Disorder-7; PHQ-9 - Patient Health Questionnaire-9; NS - not significant statistically; ${ }^{*} p<0.05,{ }^{* *} p<0.01,{ }^{* * *} p<0.001$ 
TABLE 3. The multivariate regression analyses for the total DDS-17 score and each domains score

\begin{tabular}{|c|c|c|c|}
\hline Variable & Adjusted R2 & $\begin{array}{l}\text { Regression coefficient } \\
{[95 \% \mathrm{Cl}]}\end{array}$ & $\mathrm{p}$ value \\
\hline \multicolumn{4}{|l|}{ DDS-17 score } \\
\hline Model 1 & $7.77 \%$ & $1.21[-0.08 ; 2.50]$ & 0.003 \\
\hline $\mathrm{BMI}$ & & 0.02 [0.001; 0.03] & 0.038 \\
\hline Ethnicity & & $-0.23[-0.41 ;-0.05]$ & 0.012 \\
\hline Model 2 & $26.01 \%$ & $1.66[0.49 ; 2.84]$ & $<0.0001$ \\
\hline Ethnicity & & $-0.26[-0.42 ;-0.10]$ & 0.002 \\
\hline GAD-7 score & & $0.030[0.003 ; 0.06]$ & 0.026 \\
\hline PHQ-9 score & & $0.041[0.02 ; 0.07]$ & 0.001 \\
\hline \multicolumn{4}{|l|}{ EB score } \\
\hline Model 1 & $10.07 \%$ & $0.50[-1.33 ; 2.34]$ & 0.0002 \\
\hline $\mathrm{HbA1c}$ & & 0.23 [0.10; 0.38$]$ & 0.001 \\
\hline $\mathrm{BMI}$ & & 0.03 [0.003; 0.05] & 0.028 \\
\hline Model 2 & $31.73 \%$ & $1.31[-0.32 ; 2.9]$ & $<0.0001$ \\
\hline $\mathrm{HbA1c}$ & & $0.18[0.05 ; 0.31]$ & 0.005 \\
\hline PHQ-9 score & & 0.08 [0.04; 0.11] & $<0.0001$ \\
\hline \multicolumn{4}{|l|}{ PD score } \\
\hline Model 1 & $1.28 \%$ & $1.38[0.21 ; 2.55]$ & 0.754 \\
\hline Model 2 & $4.25 \%$ & $1.71[0.50 ; 2.93]$ & 0.244 \\
\hline \multicolumn{4}{|l|}{ RD score } \\
\hline Model 1 & $10.51 \%$ & $1.32[-0.38 ; 3.02]$ & 0.0001 \\
\hline $\mathrm{BMI}$ & & 0.03 [0.01; 0.056] & 0.003 \\
\hline Ethnicity & & $-0.34[-0.58 ;-0.10]$ & 0.005 \\
\hline Model 2 & $22.50 \%$ & $1.85[0.24 ; 3.46]$ & $<0.0001$ \\
\hline Ethnicity & & $-0.37[-0.59 ;-0.14]$ & 0.001 \\
\hline PHQ-9 score & & 0.05 [0.02; 0.08] & 0.004 \\
\hline \multicolumn{4}{|l|}{ ID score } \\
\hline Model 1 & $2.99 \%$ & $1.61[-0.08 ; 3.30]$ & 0.327 \\
\hline Ethnicity & & $-0.27[-0.51 ;-0.03]$ & 0.026 \\
\hline Model 2 & $11.16 \%$ & 1.84 [0.19; 3.49] & 0.0003 \\
\hline Ethnicity & & $-0.30[-0.53 ;-0.07]$ & 0.010 \\
\hline GAD-7 score & & 0.05 [0.01; 0.08] & 0.012 \\
\hline
\end{tabular}

The table presents only significant results; for the rest of variables, there were no significant correlations noted. BMI - body mass index; HbA1c - glycated hemoglobin

on HbAlc, depression, stress, and DRD in people with both types of diabetes. ${ }^{28}$

The preliminary analysis in this study indicated a positive correlation between DRD and BMI. Overweight and obesity have been associated with low self-esteem. ${ }^{29}$ In fact, weight stigma seems to be associated with several adverse biopsychological outcomes, such as eating disturbances, depression, anxiety, body image dissatisfaction, non-adherence to medication, perceived stress, antisocial behavior, and substance use. ${ }^{29,30}$ Therefore, perhaps reducing body weight could be an important factor in overcoming psychological distress, but this needs to be properly evaluated in clinical studies.

In addition, our data suggested an association between DRD and ethnicity. Formal education or economic status did not seem to influence DRD in this study population, and the patients basically had access to the same healthcare facilities/physicians and were from same geographical area. This possibly suggests that cultural differences might impact DRD. These results seem to be in concordance with another study that indicated ethnic disparities related to psychological distress and self-care. ${ }^{31}$ Moreover, a culturally tailored diabetes self-management education and support intervention significantly improved psychological distress and self-care. ${ }^{31}$

Among the analyzed variables, the strongest positive correlations with DRD were observed with the PHQ-9 score and the GAD-7 score, respectively ( $p<0.0001$ for both). In fact, patients with clinically meaningful DRD had higher depression and anxiety scores, as well as higher prevalence of moderate/severe symptoms of depression and anxiety. This finding was similar to previous data that found significant correlations with depression (OR: $3 ; 95 \%$ CI 1.8 to $6.4 ; \mathrm{r}=0.50) .32,33$

When analyzing the four DRD domains, it resulted that emotional burden was negatively correlated with age $(\mathrm{p}=$ $0.002)$ and professional status ( $\mathrm{p}=0.011)$ in the bivariate analysis, suggesting that younger, professionally active individuals with T2DM are more prone to develop emotional distress. Our findings are consistent with a study from Vietnam, which revealed that age was negatively associated with the occurrence of overall diabetes-associated distress among DM patients. ${ }^{10}$ After full adjustment, the multivariate analysis revealed that HbAlc and depression remained important factors associated with EB. Striving to control blood glucose on the long term (and perhaps, failing sometimes) might indeed bring about feelings of being overwhelmed and fatigued, thus creating an emotional burden.

Physician-related distress was also correlated with the PHQ-9 score ( $\mathrm{p}=0.0001)$ and the GAD-7 score $(\mathrm{p}=0.001)$ in the bivariate analysis, as well as with heart rate and ethnicity. However, in the multivariate analyses, none of the correlations remained significant. It should be noted, however, that the quality of the patient-physician relationship is important for the management of diabetes, as it may influence adherence to treatment. ${ }^{34}$ Higher levels of healthcare support were shown to be associated with lower DRD. ${ }^{11}$

The bivariate analysis of our data showed that regimenassociated distress was negatively associated with age and profession, in a similar fashion as DRD, and positively with markers of glycemic control (fasting blood glucose and $\mathrm{HbA1c}$ ), as well as symptoms of anxiety and depression. Meal planning in relation to antihyperglycemic treatment, clinical and metabolic monitoring, and always follow- 
ing recommendations might be overwhelming and tiring sometimes. Previous studies in adults with T2DM pointed out that insulin-treated patients feel more distress than those on oral medication. ${ }^{10,35}$ This seems to be related rather to the burden of the insulin regimen and the challenges of reaching glycemic targets. ${ }^{35}$ Our data did not indicate an association of $\mathrm{RD} / \mathrm{DRD}$ with the injectable regimen. We have, however, analyzed oral versus injectable antihyperglycemic agents (insulin and glucagon-like peptide-1 [GLP-1] receptor agonists [RAs]), which might not imply the same level of discomfort and burden, as some of the GLP-1 RAs are administered once a week.

Interpersonal distress was associated with ethnicity and the GAD-7 score in the fully adjusted multivariate analysis ( $\mathrm{p}<0.05$ for both), suggesting that generalized anxiety and perhaps sociocultural factors are significant in creating (or easing) the ID. A previous study on 101 patients with T2DM demonstrated that higher perceived support from family members was significantly associated with lower total DRD scores. ${ }^{36}$ On the other hand, recent data also showed that not only patients with diabetes are affected by psychological conditions, but also family members (spouses) whom are at higher risk of developing depression/anxiety. The risk is driven by the severity of patient's diabetes. ${ }^{37}$

Our data basically indicated that DRD is prevalent among patients with T2DM and is associated with other neuropsychological disorders such as depression or anxiety. The nature of this interrelation is not clarified yet, therefore further longitudinal studies need to be conducted. This study also emphasizes once more the need to incorporate psychological assessments into clinical practice, and to consider the sociocultural factors when evaluating the DRD. This aspect is of importance as the condition might remain unidentified and therefore untargeted, which may have consequences on healthcare outcomes and quality of life. This also points towards a real need for integrating psychological support for patients (and their families) in the management of T2DM.

\section{CONCLUSIONS}

Psychological conditions, such as depression and anxiety, and sociocultural factors like ethnicity significantly contributed to DRD. Worse glycemic control was associated with emotional distress, possibly contributing to it.

\section{CONFLICT OF INTEREST}

Nothing to declare.

\section{ACKNOWLEDGEMENT}

The study performed in 2015 was supported by an Internal Research Grant of the University of Medicine and Pharmacy of Târgu Mureş, Romania (contract number $1 / 23.12 .2014)$.

\section{REFERENCES}

1. Cernea S, Zoltai C, Berbecilă D, Şular FL. Prevalence of Depression, Anxiety and Cognitive Impairment in Patients with Type 2 Diabetes from the Central Part of Romania. Acta Medica Marisiensis. 2016;62:209-216.

2. Naicker K, Øverland S, Johnson JA, et al. Symptoms of anxiety and depression in type 2 diabetes: Associations with clinical diabetes measures and self-management outcomes in the Norwegian HUNT study. Psychoneuroendocrinology. 2017;84:116-123

3. Lin EH, Katon W, Von Korff M, et al. Relationship of depression and diabetes self-care, medication adherence, and preventive care. Diabetes Care. 2004:27:2154-2160.

4. Zhang Y, Ting RZ, Yang W, et al. Depression in Chinese patients with type 2 diabetes: associations with hyperglycemia, hypoglycemia, and poor treatment adherence. J Diabetes. 2015;7:800-808.

5. Carper MM, Traeger L, Gonzalez JS, Wexler DJ, Psaros C, Safren SA. The differential associations of depression and diabetes distress with quality of life domains in type 2 diabetes. J Behav Med. 2014;37:501-510.

6. Fisher L, Glasgow RE, Mullan JT, Skaff MM, Polonsky WH. Development of a brief diabetes distress screening instrument. Ann Fam Med. 2008;6:246252.

7. Perrin NE, Davies MJ, Robertson N, Snoek FJ, Khunti K. The prevalence of diabetes-specific emotional distress in people with Type 2 diabetes: a systematic review and meta-analysis. Diabet Med. 2017;34:1508-1520.

8. Chew BH, Mukhtar F, Sherina MS, Paimin F, Hassan NH, Jamaludin NK The reliability and validity of the Malay version 17-item Diabetes Distress Scale. Malays Fam Physician. 2015;10:22-35

9. Zhang J, Xu C, Wu H, et al. Comparative study of the influence of diabetes distress and depression on treatment adherence in Chinese patients with type 2 diabetes: A cross-sectional survey in the People's Republic of China. Neuropsychiatr Dis Treat. 2013;9:1289-1294.

10. Nguyen VB, Tran TT, Dang TL, et al. Diabetes-Related Distress and Its Associated Factors Among Patients with Diabetes in Vietnam. Psychol Res Behav Manag. 2020;13:1181-1189.

11. Wardian J, Sun F. Factors associated with diabetes-related distress: implications for diabetes self-management. Soc Work Health Care. 2014;53:364-381.

12. Geleta BA, Dingata ST, Emanu MD, Eba LB, Abera KB, Tsegaye D. Prevalence of Diabetes Related Distress and Associated Factors Among Type 2 Diabetes Patients Attending Hospitals, Southwest Ethiopia, 2020: A Cross-Sectional Study. Patient Relat Outcome Meas. 2021;12:13-22.

13. Kintzoglanakis K, Vonta P, Copanitsanou P. Diabetes-Related Distress and Associated Characteristics in Patients With Type 2 Diabetes in an Urban Primary Care Setting in Greece. Chronic Stress (Thousand Oaks). 2020;4:2470547020961538

14. Huynh G, Tran TT, Do THT, et al. Diabetes-Related Distress Among People with Type 2 Diabetes in Ho Chi Minh City, Vietnam: Prevalence and Associated Factors. Diabetes Metab Syndr Obes. 2021;14:683-690.

15. Azadbakht M, Taheri Tanjani P, Fadayevatan R, Froughan M, Zanjari N. The prevalence and predictors of diabetes distress in elderly with type 2 diabetes mellitus. Diabetes Res Clin Pract. 2020;163:108133.

16. Kuniss N, Kramer G, Müller UA, et al. Diabetes related distress is high in inpatients with diabetes. Diabetol Metab Syndr. 2021;13:40.

17. Asuzu CC, Walker RJ, Williams JS, Egede LE. Pathways for the relationship between diabetes distress, depression, fatalism and glycemic control in adults with type 2 diabetes. J Diabetes Complications. 2017;31:169-174.

18. Schmitt A, Bendig E, Baumeister H, Hermanns N, Kulzer B. Associations of depression and diabetes distress with self-management behavior and glycemic control. Health Psychol. 2021;40:113-124.

19. American Diabetes Association. Standards of Medical Care in Diabetes. Diabetes Care. 2015;38(Supp|1):S41-S48. 
20. Wong EM, Afshar R, Qian H, Zhang M, Elliott TG, Tang TS. Diabetes Distress, Depression and Glycemic Control in a Canadian-Based Specialty Care Setting. Can J Diabetes. 2017;41:362-365.

21. Fisher L, Hessler DM, Polonsky WH, Mullan J. When is diabetes distress clinically meaningful? Establishing cut points for the diabetes distress scale. Diabetes Care. 2012;35:259-264.

22. Kroenke K, Spitzer RL, Williams JB. The PHQ-9: validity of a brief depression severity measure. J Gen Intern Med. 2001;16:606-613.

23. Spitzer RL, Kroenke K, Williams JB, Löwe B. A brief measure for assessing generalized anxiety disorder: the GAD-7. Arch Intern Med. 2006;166:10921097.

24. Johnson ST, Al Sayah F, Mathe N, Johnson JA. The Relationship of Diabetes-Related Distress and Depressive Symptoms with Physical Activity and Dietary Behaviors in Adults with Type 2 Diabetes: A CrossSectional Study. J Diabetes Complications. 2016;30:967-970.

25. Kretchy IA, Koduah A, Ohene-Agyei T, Boima V, Appiah B. The Association between Diabetes-Related Distress and Medication Adherence in Adult Patients with Type 2 Diabetes Mellitus: A Cross-Sectional Study. J Diabetes Res. 2020;1:4760624.

26. Nadine K, Ulrich AM, Christof K, Gunter W, Guido K. Reduction of HbA1c and Diabetes-Related Distress after Intervention in a Diabetes Day Care Clinic in People with Type 2 Diabetes but Not with Type 1 Diabetes. Exp Clin Endocrinol Diabetes. 2018;126:242-248.

27. Chew BH, Vos RC, Metzendorf MI, Scholten RJ, Rutten GE. Psychological interventions for diabetes-related distress in adults with type 2 diabetes mellitus. Cochrane Database Syst Rev. 2017;9:CD011469.

28. Ni YX, Ma L, Li JP. Effects of mindfulness-based intervention on glycemic control and psychological outcomes in people with diabetes: A systematic review and meta-analysis. J Diabetes Investig. 2021;12:1092-1103.
29. Wu YK, Berry DC. Impact of weight stigma on physiological and psychological health outcomes for overweight and obese adults: A systematic review. J Adv Nurs. 2018;74:1030-1042.

30. Papadopoulos S, Brennan L. Correlates of weight stigma in adults with overweight and obesity: A systematic literature review. Obesity (Silver Spring). 2015;23:1743-1760.

31. Pena-Purcell N, Han G, Lee Smith M, Peterson R, Ory MG. Impact of Diabetes Self-Management Education on Psychological Distress and Health Outcomes Among African Americans and Hispanics/Latinos With Diabetes. Diabetes Spectr. 2019;32:368-377.

32. Alzughbi T, Badedi M, Darraj $\mathrm{H}$, et al. Diabetes-Related Distress and Depression in Saudis with Type 2 Diabetes. Psychol Res Behav Manag. 2020;15:453-458

33. Chew BH, Vos R, Mohd-Sidik S, Rutten GE. Diabetes-Related Distress, Depression and Distress-Depression among Adults with Type 2 Diabetes Mellitus in Malaysia. PLoS One. 2016;11:e0152095.

34. Schoenthaler AM, Schwartz BS, Wood C, Stewart WF. Patient and physician factors associated with adherence to diabetes medications. Diabetes Educ. 2012;38:397-408.

35. Tanenbaum ML, Kane NS, Kenowitz J, Gonzalez JS. Diabetes Distress From the Patient's Perspective: Qualitative Themes and Treatment Regimen Differences Among Adults with Type 2 Diabetes. J Diabetes Complications. 2016;30:1060-1068.

36. Young CF, Shubrook JH, Valencerina E, et al. Associations Between Social Support and Diabetes-Related Distress in People With Type 2 Diabetes Mellitus. J Am Osteopath Assoc. 2020;120:721-731.

37. Nielsen J, Cunningham SA, K Ali M, Patel SA. Spouse's Diabetes Status and Incidence of Depression and Anxiety: An 18-Year Prospective Study. Diabetes Care. 2021:dc202652. 